\title{
Designing Blended Reality Space: Conceptual Foundations and Applications
}

\author{
Kei Hoshi \\ Department of Informatics \\ Umeå University \\ keihoshi@informatik.umu.se
}

\author{
Fredrik Öhberg \\ Biomedical Engineering\&Informatics \\ Umeå University Hospital \\ fredrik.ohberg@vll.se
}

\author{
Annakarin Nyberg \\ Department of Informatics \\ Umeå University \\ aknyberg@informatik.umu.se
}

\begin{abstract}
The present paper starts with a crucial discussion about the imbalance between technological and human concerns in the context of human-computer interaction, an imbalance that has arisen partly from the mechanistic aspect and its impact on interaction design. We then introduce the concept of Blended Reality Space, interactive mixed reality environments where the physical and the virtual are seamlessly combined and affect each other. The conceptual grounding and practical examples that illustrate our approach to interaction design are then discussed, adopting a standard figurative representation of blends. This helps understanding the role of blending that meaningfully bridges unbalanced separations between cognition and action, and the physical and the virtual. As a concrete example, the AGNES project, which is aimed at developing "user sensitive home-based systems for successful ageing in a networked society", is introduced. We believe that the emphasis on 'balance' or appropriate blending is very important in the development of better interactive systems for health, capitalizing on seamless combinations of the virtual and the physical in Blended Reality Space.
\end{abstract}

Keywords: Design, Theory, Metaphor, Blended Reality, Presence

\section{INTRODUCTION}

A Blended Reality Space is an interactive mixed reality environment where the physical and the virtual are seamlessly combined and affect each other. As yet, this is an ideal guiding research and design, rather than an actuality, and most mixed reality falls well short. From an idealistic viewpoint, in a true blending of the physical and the virtual, the technology itself should completely disappear from our perception. In such a situation, there will be no conscious effort of access to information (Hoshi and Waterworth, 2009; Waterworth and Waterworth, 2010). It would then be possible to realize an ideal in which our activities are characterized by a natural flow of action, without any intrusion from technology arising from the physical-virtual divide. The user would perceive and act directly, as in everyday life unmediated activities.

\section{ISSUES OF INTERACTION DESIGN}

\subsection{Cognition and Action}

Rene Descartes established a period of thinking about cognition that separated mind and matter, cognition and action. There is, by this view, the 'separated environments' assumption: the idea that there are two separated environments, a mental (subjective) world and a physical (objective) world. The revolution of digital computers largely relies on mechanical metaphors. It seems that technological mastery has accrued much of the prestige of science, rather than its origin of scientific thinking. Many recent scholars have argued against this, for example, as the mind-as-machine view by George Lakoff (1987) and as the mechanistic picture of the world by Dijksterhuis (1965).

Human Computer Interaction 'Design' largely relies on the mind-as-machine view (Lakoff, 1987) or the mechanistic picture of the world (Dijksterhuis, 1965). It aims to practically harmonize accessibility, usability, acceptability, and engagement based on a formalization of human cognition of the world. This equals avoidance of the complicatedness of the real world and of humans. This issue has long been discussed in the field of cognitive science and psychology (see also Reed, 1996). 'Design' has been conceived as 'scientific' to the extent that it has kept away from dealing with everyday concerns. The mechanistic view tends to reinforce the preconceived ideas that;

1) to the extent that it is scientific, design must be about bodies as distinct from minds;

2) to the extent that it is about minds, design cannot hope to be scientific in the explanatory sense.

Traditional $\mathrm{HCl}$ researchers have the assumption that the brain functions to construct and utilize representations of the world around us, 'a model of the world' (Craik, 1943; Reed, 1996). The animal must collect, collate, and interpret stimuli until it has a 'model of the world' constructed by the brain or mind, in order to let it send commands that will cause its body to behave in suitable ways. Several scholars and disciplines have argued the limitation of this cognitivist view of $\mathrm{HCl}$, as found in discussions in terms of augmented and mixed 
realities, tangible interaction, and situated action (e.g. Dourish, 2001; Suchman, 2007).

George Lakoff (1987) refers to mind as a computer with biological hardware and has contended that "the mind runs using programs essentially like those used in computers today and it may take input from the body and provide output to the body, but there is nonetheless a purely mental sphere of symbolic manipulation that can be characterized in terms of algorithms of the sort used in computer programs." Such mechanical systems all have one thing in common: They do not act unless put into action by an external agency. Based on the assumption, it may be true that a tool is something that extends the action of workers. Therefore a tool, for example a computer, can do this only because workers and other sources of power bring it into action. Designers of interactive systems have tended to assume that every manifestation of behaviour requires some kind of 'stimulus' either from outside the system or from inside. There are so-called reactive mechanisms based on external stimuli and instructive mechanisms based on internal stimuli or commands in psychological theories (Reed, 1996). While it may be true of machines, and of robots as we know them, that a stimulus is needed to bring them into action, it is far from true of animals. Animals are always active in whole or in part. And even though machines, tools and computers are not active in the way that animals are, interactive systems have been designed on the basis of modelling animal and human behaviour on mechanical principles.

Humans have to adapt their behaviours to the way computers do things, and in which no such constant activity occurs. The whole idea of $\mathrm{HCl}$ still largely deals with the complex environmental conditions in which humans put themselves into motion via stimuli or inputs signalled to the brain. Their behaviours as responsive outputs commanded by the nervous system. While the computing environment has become more intelligent and pervasively penetrated into our everyday live, is it suitable for humans?

We can find challenging and interesting applications for educational, medical and industrial usage designed on the basis of mechanical principles, but many are not at all suitable, and especially not for people with special needs. Human beings have to forcibly adapt to the computing environment based on mechanistic principles, even though human behaviours should be essentially a natural flow of action based on constant activity.

\subsection{From mixed to blended}

Technology creates the virtual world, but also exists in the physical world - with which the virtual often competes for our attention. Many new interaction styles clearly exhibit the combination of the physical and the virtual, sometimes called mixed reality (Jacob et al., 2007; Chalmers et al., 2003; Rogers et al., 2002). However, there are several physical constraints that limit users' interactions, such as those of physical displays, other input-output devices, and social factors. For example, the user typically concentrates on foreground tasks through full access to a fixed display. The mouse reflects the two dimensional paradigm that supports easy spatial navigation, by clicking icons, selecting areas and dragging representations of data objects. Although a flexible approach, this two-dimensional input-output interface is still limited when applied to, for example face-to-face collaboration or distributed environments. Most people struggle with using the computer and embedded systems. Further, the elderly and socially handicapped have become increasingly dissatisfied with the environment or disadvantaged by it. Hoshi and Waterworth (2009) have experimented with the influence of blended reality space, and Hoshi (2011) discussed the need for a change from mixed to blend, especially for the vulnerable, the elderly and the socially handicapped.

Today, mixed realities of different kinds represent an increasingly prevalent approach to interaction that strives to combine the physical and the virtual. Mixed reality is also a growing object of study for the $\mathrm{HCl}$ research community, as part of a widespread effort to develop viable and more flexible alternatives to WIMP-based GUIs. Most of the broad range of new interfaces developed by $\mathrm{HCl}$ researchers are seen as alternatives to the current GUI paradigm and try, in one way or another, to diverge from the WIMP-based approach (Jacob et al., 2008). For example, sensor-based techniques for interacting with virtual entities via the manipulation of physical objects in space have been explored by $\mathrm{HCl}$ researchers.

Recently, we have witnessed the emergence of a wider variety of $\mathrm{HCl}$ technologies, such as those implemented within sensor-based environments, handheld smart phones with more intuitive onscreen interfaces and orientation sensors, and these and others are now gradually penetrating society. They emerge as a huge growing trend in the $\mathrm{HCl}$ literature. Typical examples are; augmented reality, tangible interaction, ubiquitous and pervasive computing, context-aware computing, handheld, or mobile interaction and so on (Jacob et al., 2008). Since the ubiquitous GUI was introduced and became the standard paradigm in $\mathrm{HCl}$, the post-WIMP trend is that digital media are becoming more pervasive in our everyday life. Everywhere in our built environment devices such as video screens, electronic access systems, and sensor-based smart environments are rapidly increasing. But there is still a huge gap between the media and ourselves as bodies in physical space.

We still do not have a clear understanding of the scope of this phenomenon, especially its perceptual and psychological aspects. We predict that the intersection of sensory, cognitive and emotional aspects in emerging mixed realities will be significantly important in attempts to go a further step in the development of better combinations between the physical and virtual environment, in what we call Blended Reality Spaces (Hoshi and Waterworth, 2009). The history of $\mathrm{HCl}$ is can be seen as the evolution of the standard WIMP interface composed of desktop metaphors. When 
metaphors don't work well, they might lead to people to develop inappropriate expectations of technologies (Imaz and Benyon, 2006). In order to understand how blends are framed and formed, we need to get the picture of metaphors. Metaphors and blends actually work together. It helps to also understand the embodied notion in $\mathrm{HCl}$, derived from our bodily and social experiences.

\section{METAPHORS AND BLENDS IN HCI}

\subsection{Metaphor}

We usually don't have a doubt that metaphors have been used in the design of digital devices. The well recognized personal computer user interfaces have been designed on this basis - known as the 'desktop' metaphor. The designer explicitly tries to draw on people's knowledge of office work to help them understand the operation of the computer. The traditional approach to human-computer interaction $(\mathrm{HCl})$ design uses metaphor to communicate the functionality of the system to the user (Imaz and Benyon, 2006), which encompasses what users feel, think, and are able to do as they interact with the products/systems that deal with the formalization of human cognition of the world. The designer draws on users' experience in another domain to assist their understanding of the system (Waterworth et al., 2003). Over the past twenty years, more and more interfaces have adopted this style, and now it is spreading to other devices such as mobile phones, digital cameras, audio-visual equipments, and many web sites in our everyday life. The metaphors should work well where the designer and the user perceive them in similar ways. But while metaphors are provided to let people bring their previous experiences to understand new interactions, they might lead to people developing inadequate expectations of technologies. Much of this confusion arises because metaphor is not well understood. That means that the embodied notion of metaphor, derived from our bodily and social experiences, is not understood well. In order to gain insight into a metaphor that structures the action we perform in a particular culture, Lakoff and Johnson (1980) refer to "a culture where arguments are viewed in terms of war".

\section{"ARGUMENT IS WAR"}

According to Lakoff and Johnson (1980), people in the culture understand, feel and act the battle with their body. In the culture, arguments are partially structured by the concept of war such as an attack, defense, shoot, etc. This is a linguistic battle, of course, not a physical battle. If there is a culture where arguments are viewed in terms of 'dance', they have a discourse structured in terms of 'dance'. We can assume that people in this culture similarly understand and experience argument in terms of dance performance. As Lakoff and Johnson (1980) mentioned, they live in a culture where in arguing no one wins or loses, there is no sense of attacking or defending. Human thought processes are largely metaphorical, and we act according to the way we imagine things, which can vary from culture to culture because metaphors are rooted in physical and cultural experiences (Lakoff and Johnson, 1980). Clearly, there is a gap to be bridged by an intelligent effort on the part of the person to whom we want to tell what a word means. Michel Polanyi (1966) has pointed that "our message had left something behind that we could not tell, and its reception must rely on it that the person addressed will discover that which we have not been able communicate". This example illustrates how there can be significant misunderstanding or gaps even in the same or similar cultures. In every culture, linguistic metaphors are tools for understanding and can be meaningful and true. But the embodied gap exists somewhere between different cultures (Lakoff and Johnson, 1980). The gap between designer and user is, in most cases, essentially a bodily, crosscultural gap. In designing interactive systems for healthcare, the gaps can be found in several places, for example between patients and doctors, or elderly people and care givers.

We feel our physical experiences of the world, our spatial awareness, our bodily movement and the way we manipulate objects, through metaphors. Imagine our everyday life, we adopt a drooping posture when we come upon sadness or are living with depression. On the other, we adopt an erect posture when we feel positive emotional states. We sometime describe our feelings as "feeling up" or "feeling down" to understand indefinite substance in terms of directionality (Lakoff and Johnson, 1980). Lakoff and Johnson mentioned the fact that "we have bodies of the sort we have and they function as they do in our physical environment". Spatialization such as this shows that the way we think, what we experience, and what we do every day is very much a matter of metaphor. Metaphors are pervasive in everyday life, not just in our discourse but in our thought and action.

"Argument is war", spatialization and other metaphors take account of an embodied prelinguistic structure of experience that motivates conceptual metaphor mapping, called an 'image schema.' According to Lakoff (1987), image schemas are simple structures that constantly recur in our bodily experience, formed from our bodily interactions, from linguistic experience, and from historical context. Schemas have been applied in 'experiential' approaches to design. For example, Waterworth (1999) developed 'scheme space,' an interactive system that seeks to capture the human scale of people interacting with basic-level categories, and through which interaction is most natural both conceptually and perceptually (see also Waterworth et al., 2003).

\subsection{Blend}

As Lakoff and Johnson (1980) described it conceptual metaphor refers to the understanding of one idea or conceptual domain, in terms of another. It is a cross-domain mapping, taking elements from one domain and applying them to another (Figure1). 
Figure 1: Cross-Domain Mapping and Cross-Space Mapping (based on Imaz and Benyon, 2006)
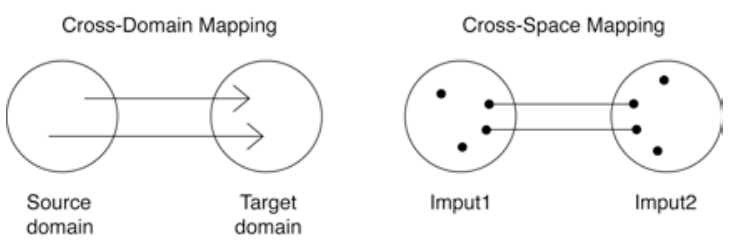

The conceptual domains hypothesized in conceptual metaphors have two main roles: source and target. For example, English expressions like

"My computer is a desktop", which implies a desktop metaphor of a PC.

"Desktop" is source domain from which we bring metaphorical expressions (e.g., My computer is a desktop).

"My computer" is target domain that we try to understand and experience (e.g., My computer is a desktop).

According to Imaz and Benyon (2006), "if the metaphor is a cross-domain mapping, taking elements from one domain and applying them to another, then blending is an operation that is applied to two input spaces, and which results in a new, blended space". Blending is the ability to take two mental spaces, and connect them in certain ways such that a blended mental space emerges, and this is also the ability that gave rise to art, science, and language (Fauconnier and Turner, 2002). The concept of mental space refers to partial cognitive structures that emerge when we think and talk (Fauconnier, 1997). According to Fauconnier (1997), there is a process of mental construction where background experience and cultural history are brought to play in the form of frames or schemata, in between language and the real world. Mental spaces are set up and built on through many sources. 'Connectors' link mental spaces to one another (figure 1). A mapping between an element of one space onto one or more elements of another is established by a connector. As shown in figure 1 using standard figurative representations originally used by Fauconnier and Turner (2002), connectors map elements from both spaces, a source mental space and a target mental space. Mental spaces are established, structured, and linked to other spaces (figure 1). Blending works as follows (Figure 2):

1) generic space: reflects abstract structure andorganization shared by the inputs, and defines the core cross-space mapping between them.

2) cross-space mapping: elements and relations between two input spaces are connected.

3) blend: it is a new emergent structure not provided by the inputs.

Conceptual metaphor and conceptual blending are about the idea of projection of structure between domains. As blend theorists such as Imaz and Benyon (2006) point out, there are significant differences between the approaches as follows.

Whereas conceptual-metaphor studies relationships between pairs of domains, conceptual-blending establishes a network of spaces, usually four.

The conceptual metaphor view considers the metaphor as a directional phenomenon from the source domain to the target domain, as shown Figure 1, while conceptual blending considers projection going from any mental space to any other as shown Figure 2.

Figure 2: Generic space and Blended space (based on Imaz and Benyon, 2006)

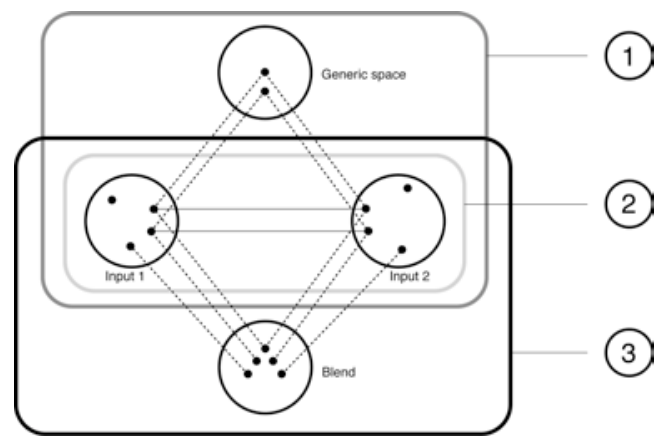

Whereas the conceptual metaphor view seeks everyday life-based discourse and knowledge, which is embodied conceptual relationships between domains, conceptual blending is focused on a new conceptual domain. Especially, the mental space in blending is the basic unit of cognitive organization but the conceptual metaphor view focuses on a solid relationship between two conceptual domains. More importantly, since conceptual blending is focused on new conceptualization, the newly emergent space is often different from the real world we normally experience. The gap between a user and an interactive system, when users still struggle to use or understand newly released systems even though technologies have been evolving steadily, is caused by this issue.

\subsection{Desktop Interface as Blend}

The WIMP-based GUls have become a blend rather than a metaphor, since the notion has become a new emergent space (2006) - a thing in itself as far as cognition is concerned. Figure 3 shows how blending works with the PC desktop.

Figure 3. PC desktop as blend

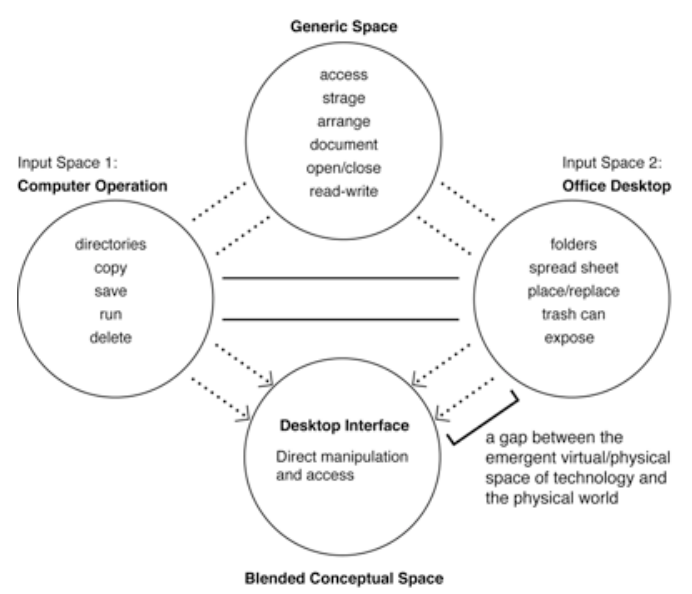


(i) Two input spaces exist, input space-1, input space-2 and the solid lines indicate a crossspace mapping that connects elements and relations between the inputs. The two principal inputs have different organizing frames. Input space-1 refers to the frame of traditional computer operations, and input space-2 refers to the frame of office work. The dotted lines refer to connections between inputs and either generic or blended space.

(ii) A generic space maps onto each of the inputs and contains what the inputs have in common, which reflects some more abstract structure and organization shared by the inputs.

(iii) Blended conceptual space is an emergent conceptual structure with new ideas and insights. The emergent property of the blend provides direct manipulation and access.

In the interfaces using the 'desktop metaphor' with direct manipulation and access, the grasping, releasing, and opening of an object are imitated by dragging, dropping, and double clicking on perceivable icons, objects and folders on the virtual surface. These are new emergent functions that exist neither in the real world nor the domain of computer operations. They appear in the blended conceptual space. Because of this newly emergent space, the experience is very distinctive from physical experience in everyday life.

We can clearly experience the gap between the new blended space and our physical world of action. For example, as Imaz and Benyon (2006) suggested also, a computer window in the blended space is different from a real window and a menu on the space is different from a menu in a restaurant. Therefore, users often encounter a physical-virtual gap that disrupts the flow during activities that require a changeover between the physical and the virtual. They are forced into conscious effort to access information and carry out intentions.

In the process of designing $\mathrm{HCl}$, applied user observation techniques based on working more closely with users have been introduced, which include qualitative research techniques such as ethnographic studies, participatory design that directly observes users' behaviour and the environment across a number of people who use products, who tell stories as users, who use a particular product. However, designers following the human-centred principles have often designed complex, confusing systems. In fact, while the usability, accessibility and understandability of the systems have been improved, the complexity of the products still remains high. There exists an embodied gap between the designer and the user.

\subsection{The Notion of Blended Reality Space}

Hoshi and Waterworth (2009) have discussed issues relating to presence arising from the recent evolution of tangible interaction techniques as an alternative interaction paradigm to the familiar WIMP-based Graphical User Interfaces (GUIs).
They introduced the concept of Tangible Presence in Blended Reality Space, and its study as an emerging weaving of $\mathrm{HCl}$ and presence research. According to their discussion, The theoretical notion of Blended Reality Space arising from blend theory was not discussed, but an experimental study was described which examined the influence of factors in the way blended realities may be implemented. They have identified notable attributes during free movement-based game activities in blended reality space, namely tangibility and sense of presence.

On the basis of blend theory, Blended Reality Space are described as Figure 4 that shows a new emergent experience space that is immersive, interactive and body-movement oriented, and where there will be less or no conscious effort of access to information. The user perceives and acts directly, as in everyday life unmediated activities. We see the first examples of this in some commercial games that have been applied to training people with sensorimotor disorder or with cognitive dementia. In typical examples of both Nintendo's Wii ${ }^{\mathrm{TM}}$ (Nintendo Inc., Kyoto, Japan)based and some video-capture games, the players have no direct physical connection with the game environment. Their physical movements are detected by either the 'Wiimote' (the Wii remote control) or by a camera. Body movements performed by players are generally in response to game-initiated events. When their free body movements in physical space are tracked and used as inputs to the game, a truly merged physical/media space may be created during play, a clear example of Blended Reality Space.

Figure 4. Blended Reality Space

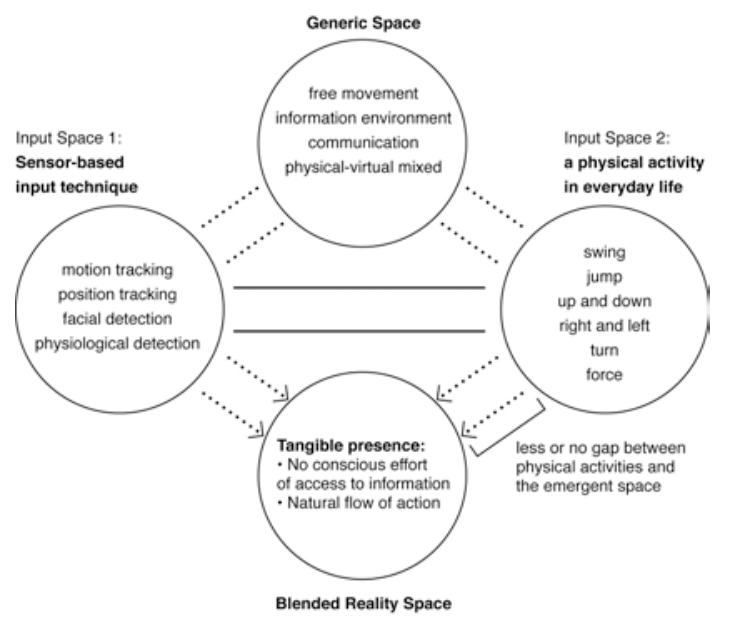

The feasibility of using the Wii-system in a rehabilitation setting for adolescents with cerebral palsy has been evaluated in a case study (Deutsch, 2008). This example shows the potential of blended reality space for people with special needs. This interaction style is formed in the harmony between the physical and the virtual, utilizing tangible interaction. In a true blending of the physical and the virtual there will be no gap between the emergent virtual/physical space of technology and the physical world (figure 4). True blended reality space will release human actions from physical constraints and physical-virtual disruption, and 
provide natural flow of action, equivalent to those in the physical world. The nature of this Blended Reality Space can be expected to also produce a strong psychological feeling of presence (the perceptual illusion of non-mediation (Lombard and Ditton, 1997) within the merged space, since the technology effectively disappears from attention. As media becomes increasingly interactive, perceptually realistic, and immersive, the experience of presence becomes more convincing (ljsselsteijn and Riva, 2003). However, since information and communication technology become more pervasive in the built environment, such as video screens, electronic access systems and smart sensor techniques, the effectiveness of interactive mixed realities has been linked to the sense of presence as judged by users of the space (Bernardet et al., 2008). What does blended reality space contribute to the experience of presence?

The presence parameter may vary according to several factors, including the available technology, the required use, and the context of use. Effective interactive systems for communication will work by creating harmony through using appropriate contexts and thus create optimal presence states. Also the contextual cues are expected as material, shape, size, texture, and weight configuration of the physical object. Haptic feedback helps users feel a degree of tangibility, a convergence between the physical and virtual. But while haptic feedback can contribute, tangibility is more than just haptics.

In a Wii tennis situation, using a racket is the natural way of playing tennis, the player has already became well practiced and experienced without explicit user manuals or oral guidance. Normally, the user of such embedded devices as mobile phones, computer applications, and medical devices needs an early stage of learning to use a tool/device, performed with conscious attention (Kaptelinin and Nardi, 2006). The physical racket fills the gap between the physical and the virtual world. The conditions of weight, texture, and size of the racket, combined with sensor based interaction techniques, help in increasing the experience of "being there" in the blended reality space. The quality of actions and the strength of presence are correlated (Hoshi and Waterworth, 2009). The feeling of presence provides to the subject feedback about the status of its activity within the situation in which the activity is carried out (Riva, 2005).

The main idea of such a tangible interface, built on movement and position sensing techniques, is to provide physical forms which serve as both representations of and controls to digital information. The applications make the digital information directly manipulable with our hands, and perceptible through our peripheral senses through their physically embodiment (Ullmer and Ishii, 2000; Ishii, 2008). It is vitally important that the emerging trend towards tangibility is provided using the most appropriate combinations of the physical and virtual. Optimal combinations of tangibility and evoked presence carry the potential to make full use of, while not overburdening, the flexible but limited capacities of selective attention; this will be a key issue for the design of future interaction approaches, what we call Tangible Presence in Blended Reality Space (Hoshi and Waterworth, 2009).

\section{THE CASE OF AGNES}

Figure 5 is a schematic outline of our approach: a context-sensitive home-based interactive system, in which humans utilize background information with ambient media by means of a tangible object but without being disrupted in their foreground tasks. This system is composed of ambient displays, tangible interaction objects and interaction mechanisms and protocols, including gesture detection, which makes for easy-to-use and natural interaction. The tangible object and the ambient display complement each other and provide suitably gentle notifications and other information, and establish communication with connected persons. The human focusing of attention between background and foreground has to be a smooth transition, which makes it possible to achieve a natural flow of actions without awareness. By using commonly available and cost effective technologies such as cameras, motion sensors, and mobile devices, the system uses suitable algorithms to classify body pose and methods to associate the extracted pose information over time with gestures by detecting the users' states and activities without any use of intrusive sensors (Waterworth, 2009). Elderly people living at home will use the system actively by accessing information, sending messages or requesting services.

Figure 5: A context-sensitive home-based interactive system

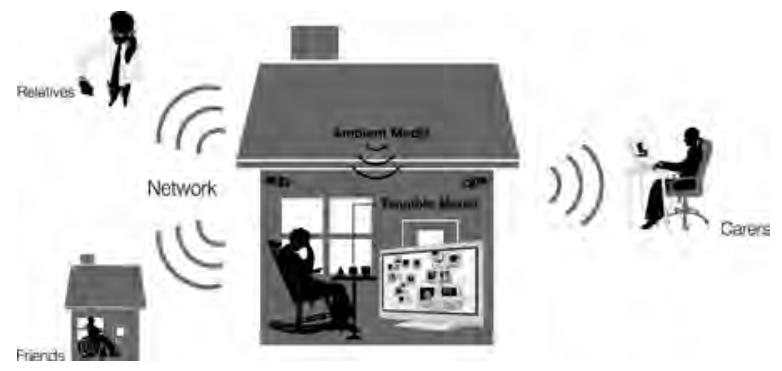

\subsection{Ambient and tangible interaction in AGNES}

In AGNES, an ambient display combined with a tangible object in the context of a person's home form a Blended Reality Space, comprising a radically new way to manage social interaction.

\subsubsection{Ambient Display in AGNES}

In everyday life, we pick up natural sources of ambient information to understand how things are around us. For example, people could experientially interpret implicit information from outside the window. A subtle combination of brightness, wind direction and humidity gives us the feeling of the coming rain. The perceptual feeling of a peaceful curtain-wave makes people placid, or people foresee a storm when they see the curtain waving in the dim light of the window, with no explicit information or conscious effort. 
The AGNES system will generate a variety of ambient media such as sound, light, airflow, colour as background interfaces to virtual space at the periphery of human sensory perception (figure 6). The elderly person connected to others through the social networking technology receives information such as messages or stories from family members and others transformed into a variety of ambient forms through an $1 / O$ unit. For example, the combination of subtle wind and green LED implies notification coming from family members, mid-level breeze and red LED gives important information from them, and strong wind and vivid red LED indicates an urgent message from them.

Figure 6. Ambient display in AGNES

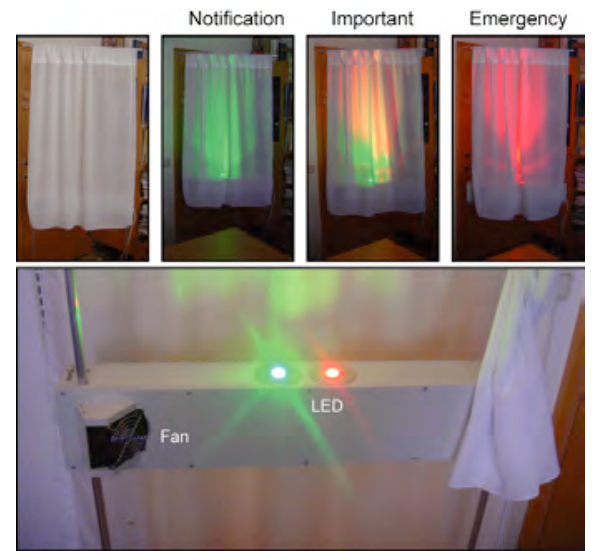

The ambient display is built on the basis of the blending framework previously discussed on the present paper (figure 7). First, there is a crossspace mapping consisting of two conceptual inputs: input-space 1 is explicit information with current technology-based information displays such as voice/text messages, state-activity reports and notifications. The other, input-space2, is natural sources in everyday life with their sounds, light, airflow, breeze and shadow. Second, the generic space implies some more abstract structure shared by the inputs. Third, blended reality space, a fourth space, is a new emergent structure that provide tangible presence with no conscious effort of access to information.

Figure 7. Ambient display as blend

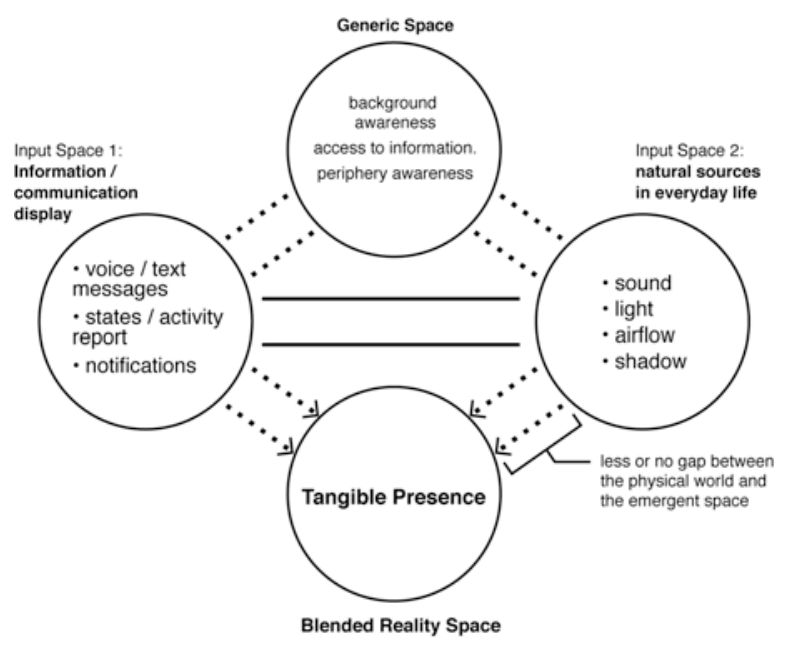

4.1.2 Tangible Objects in AGNES

Imagine a situation in the physical world, where an elderly person needs to ask questions of his or her relatives or neighbours. The elderly person may lay a hand on the relative's shoulder, or may knock on the neighbour's door. If it is an emergency situation, they may strongly tap relatives or may knock the door severely. The contextual cues on the surface of the AGNES tangible object provide access to implicit memory (figure 8). The object affords tapping to contact a loved one. We need to carefully choose contextual cues suited to various situations, wood texture to afford knocking, boa material to afford stroking and knit material to afford tapping, based on everyday life objects (figure 8).

Figure 8. Tangible Object in AGNES

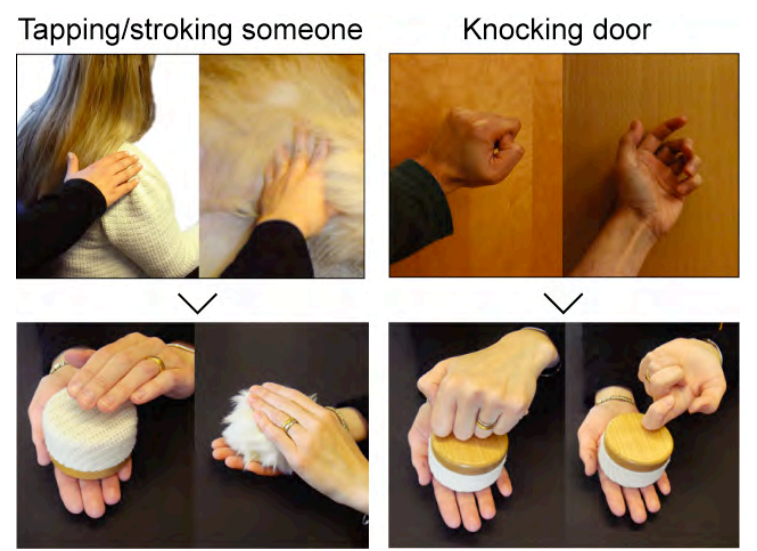

The tangible object is built on the basis of blending frameworks as well (figure 9). Input-space1 is that of sensor-based information input techniques such as motion tracking, position tracking and physiological detection, for example. The other, input-space2, is physical activities in everyday life such as tapping, shaking, knocking, and stroking.

Figure 9. Tangible Object as blend

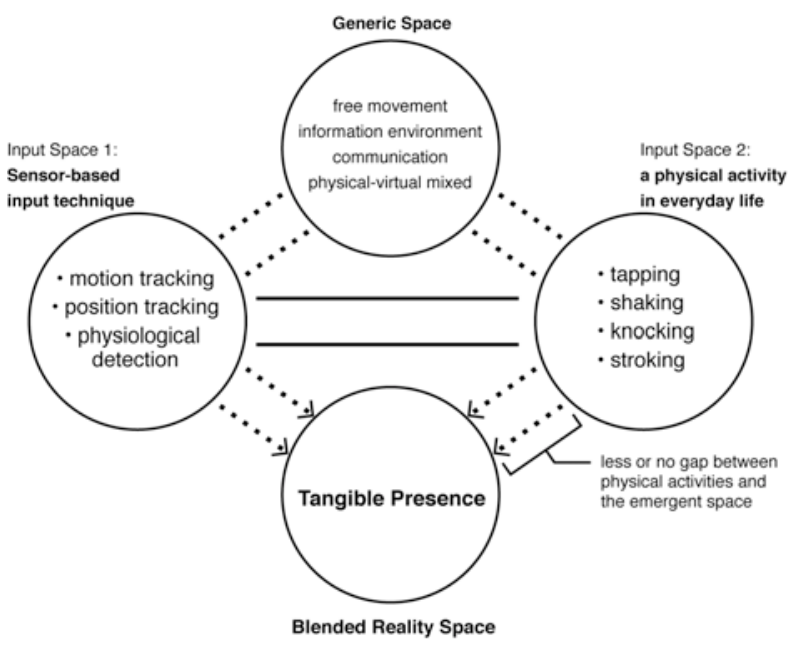

Here, literal and figurative expressions are established.

"Information is breeze from a window"

"Communication is tapping someone (memory)" 
The expression helps understand information and communication in terms of natural sources/physical activities in everyday life, where there is some similarity or correlation between them. Users understand meaning in their bodily interaction with the world, physically, socially, and culturally.

The elements of the input spaces in both the tangible object and the ambient display are chosen on the basis of bodily knowledge in everyday life. These are especially suitable for elderly people who lose explicit and abstract knowledge, but still behave efficiently from their embodied knowledge. Our experiential design for interaction focuses on human beings having the same primitive experiences covering our common/shared embodied knowledge, and this is an approach to designing applied in our everyday live, in ways that are implicit, often unconscious, not easily externalisable in words, which only our bodies know (Hoshi, 2011). This is on a more human scale, more experientially sensitive and aesthetical, and more modestly disappearing from our awareness.

\section{INVOLVING USERS}

In an initial trial, qualitative interviews with three different focus groups, in total 25 volunteer users between the age of 65 and 94 and living in their own homes, have been conducted in Sweden. Some of the users live alone and manage their everyday activities without any help. Others, who are suffering from various stages of dementia, live with partners. Further, other users, who suffer from aphasia, live with partners that help them, but some of them live alone. The focus group interviews were divided into two meetings and each meeting lasted for about 90 minutes. The meetings were audiotaped and documented with field notes. The first meeting with each group focused on introducing the AGNES project, especially on its aim to conduct user driven technology development. The conducted discussions were focused on information technology in their everyday activities and the potential to increase safety, to counteract loneliness and to increase social activities. In the second meeting, our prototypes, the ambient display and tangible object, were introduced. The basic ideas of the design were described by demonstrating video-prototypes for the volunteer users. The demonstrated scenarios helped their understanding of how the technology could potentially be used and served as valuable starting points for the discussions.

\subsection{The functionalities}

The limited functionality and simplicity of the ambient display and the tangible object were appreciated by the volunteers. They commented on the fact that the tangible object might be lost, what might happen if they would drop it and it might roll away. From this, they discussed alternative shapes that would be easier to grasp and would not roll. For example, the users mentioned the stiffness in their hands. Shapes like kidneys or dog bones were mentioned as good for grasping with their hands. They were also concerned about the fact that they must stay at home in order to get the messages/information. They were afraid of missing some important notifications if they were out. They suggested other ways based on everyday objects that they would be able to take with them when they left their homes, such as bracelets and keys, which seemed both discrete and difficult do leave behind. They also considered the need for complementary use of the prototypes and the telephone and the wrist alarm that some of them have been using already in their everyday life.

\subsection{The meaning}

The material aspects of the tangible object, such as wood and soft fabric, were appreciated. In contrast to using current mobile phones, such simplified interaction methods as tapping and knocking were for the most part appreciated by them. All seemed to be very curious about the ambient display. They seemed to like the lights and breeze. They questioned whether it would be possible to differentiate the various colors from one another. Sound as an alternative was put forward. Their comments also suggested that the ambient display should be installed in an appropriate location at their home for them to effectively sense and recognize the implicit information conveyed. The quality of fabric material for displaying the implicit information is very critical in order to let them use their sensory perception. Their comments urged us to reconsider appropriate contextual cues, such as suitable shape, material, color and even breeze. User-led innovation is a key part of our design approach. We will conduct further user studies to investigate our improved prototype in order to effectively match the needs of our target users.

\section{DISCUSSION}

Our target users, elderly people, some with mild cognitive impairment and dementia, normally have difficulties dealing with explicit knowledge and memory such as understanding texts, making inferences, encoding information into memory, and retrieving information from memory. Contrarily, recent notable successes to improving cognitive function works by exploiting intact cognitive process such as implicit memory (Ballesteros et al, 2009), revealed by performance effects in the absence of deliberate recollection. Elderly people are still capable of using and being influenced by their past knowledge, whether they are be aware of it or not (Zacks, 2000). This is an automatic and unconscious form of memory. They have knowledge that their muscles physically remember, but explicit sources of knowledge such as user's manual, verbal assistance, and so on, are unsuitable for them. Designing simple and adequate representations for peripheral media using tangible objects is a key part of developing better combinations of the physical and virtual. The tangible object in the system plays a role that wakes up implicit memory in which previous experiences supports the performance of a task without conscious awareness of these previous experiences (Hoshi, 2011). It has to be designed to link an everyday object and activity that humans bodily remember. 


\section{CONCLUSION}

The unifying idea within the present paper is its approach to the subject matter of 'design'. Several researchers have previously argued that a freebody movement based interaction is still inconsistent. For example, Chalmers et al., (2003), Broll and Benford (2005) have questioned the idea of seamless interaction with ubiquitous computing as undesireable. No mixed reality environment can ever be completely seamless, but for some users and situations, maximum seamlessness is a necessity. Our prototypes with embodied interaction are first steps towards the creation, manipulation, and sharing of meaning through interaction nicely employed within Blended Reality Space in the service of elderly users.

The appropriate combination between action and meaning is about the relationship between tangibility and evoked presence, which are incorporated into a practice. The action and meaning are not opposed, but engage each other. Our concept is based on the assumption that action and cognition, mind and body are not dissociable, but affect each other (see also Dourish, 2001).

We have argued that humans are not machines that need stimuli or input signaled to let them take action. It should not generally be the case that we are aware of something, in the here and now, and then pick up an object and act. This is mostly quite unnatural and implies explicit knowledge and conscious effort. Rather, when functioning smoothly, we act without conscious awareness, and then find later that we have been using a tool. The ambient display and the tangible object are not the stimulus that provokes an action. Modestly informed by peripheral information and unconsciously executed, they restore the primacy of action and re-integrate the mind and the body. The technology then disappears from perception in use, with no conscious effort of access to information or effort of interaction. Although current technology cannot be embedded as a perfectly consistent and seamless blend, we believe that our approach should be suitable for people with special needs, especially those who have difficulties dealing with explicit knowledge, as discussed previously in the present paper.

Our concept, an emerging integration of $\mathrm{HCl}$ concerns and Mediated Presence research, Tangible Presence in Blended Reality Space, bridges the contextual reality gap in the sharing of meaning through mediated communication within the blended reality space. Tangible Presence in Blended Reality Space is based on the integration of metaphor theory, blending theory, design and presence that have one important matter in common, 'embodiment', within the domain of everyday life based people, behaviours, objects, and their interaction with information and communication technologies. We have sought to re-integrate separated environments as an attempt to approach the subject matter of design (see section 2.1). Conceptual blending has emerged in the last decades with new advances and refinements, and is based on the integration of cognitive semantics, metaphor theory and blending theory (Imaz and Benyon, 2006). We should not ignore the complicatedness of the real world and of humans, involving the organism, action, and the environment, as Dourish (2001) pointed out. This insight is rooted in Gibson's (1986) ecological perspective that "Ecological psychology studies knowledge in the world rather than knowledge in the head."

We believe our integration is a new way of approaching to $\mathrm{HCl}$ design, what we would call "Human-Experiential Design" (Hoshi, 2011). This approach represents an important step in the development of better e-health, capitalizing on seamless combinations of the virtual and the physical in blended reality. Significantly, an experiential approach to design incorporates bodily experiences into developing interactive systems, and true universal design for everyone becomes possible. This is because all humans - including elderly people and the disabled - have the same primitive experiences arising from common and shared embodied knowledge.

\section{ACKNOWLEDGMENTS}

The authors acknowledge the contribution of Prof. John Waterworth in the many discussions we have had on the topic of this paper, and of all AGNES partners and volunteer users in the project work. AGNES is funded under the Ambient Assisted Living (AAL) Joint Programme, a new joint research and development (R\&D) funding activity implemented by 20 European Member States and 3 Associated States with the financial support of the European Community based on article 169 of the EC treaty.

\section{REFERENCES}

Ballesteros, S., Gonzalez, M., Mayas, J., Reales, J. M., \& Garcia, B. (2009). Crossmodal object priming in young and older adults: Multisensory processing in vision, touch, and audition. European Journal of Cognitive Psychology, 21(Issue 2, 3), Aging, cognition, and Neuroscience, 366-387

Bernardet, U., Inderbitzin, M., Väljamäe, A., Mura, A., \& Verschure, P. FMJ. (2008). Validating Presence by Relying on Recollection: Human Experience and Performance in the Mixed Reality System XIM. In A. Spagnolli, \& L. Gamberini (Eds.), Proceedings of the 11th International Workshop on Presence (pp. 178-182). Padova, Italy.

Broll, G., \& Benford, S. (2005). Seamful Design for Location-Based Mobile Games. In ENTERTAINMENT COMPUTING - ICEC 2005, Lecture Notes in Computer Science, Volume 3711 , 155-166

Chalmers, Matthew., \& MacColl, Ian. (2003). Seamful and Seamless Design in Ubiquitous Computing. In Proceedings of Workshop At the Crossroads: The Interaction of $\mathrm{HCl}$ and Systems Issues in UbiComp.

Craik, F. J. W. (1943). The nature of explanation. London: Blackwell

Deutsch, J. E., Borbely, M., Filler. J., Huhn, K., \& Guarrera-Bowlby, P. (2008). Use of a low-cost, commercially available gaming console (Wii) for 
rehabilitation of an adolescent with cerebral palsy. Physical Therapy Journal, 88(10), 1196-207.

Dijksterhuis, E. J. (1965). The mechanization of the world picture. Oxford: Clarendon Press.

Dourish, P. (2001). Where the Action Is: The Foundation of Embodied Interaction. Cambridge, MA: The MIT Press.

Fauconnier, G. (1997). Mapping in Thought and Language. Cambridge: Cambridge University Press.

Fauconnier, G., \& Turner, M. (2002). The Way We Think: Conceptual Blending and the Mind's Hidden Complexities. New York: Basic Books.

Hoshi, K., \& Waterworth, J. A. (2009). Tangible Presence in Blended Reality Space, PRESENCE 2009. Proceedings of the 12th Annual International Workshop on Presence. Los Angeles, CA, USA.

Hoshi, K. (2011). Reframing Dichotomies: Human Experiential Design of Healthcare Technologies. In Ziefle, M \& Röcker, C (Eds.), Human-Centered Design of E-Health Technologies: Concepts, Methods and Applications.Hershey, PA, USA: IGI Global.

ljsselsteijn, W., \& Riva, G. (2003). Being There: The experience of presence in mediated environments. In G. Riva, F. Davide \& W.A. ljsselsteijn (Eds.), Being There: Concepts, effects and measurement of user present in synthetic environments (pp. 3-16). Amsterdam: IOS Press.

Imaz, M., \& Benyon, D. (2006). desining with blends: conceptual foundations of human-computer interaction and software engineering. Cambridge, MA: The MIT Press.

Ishii, H. (2008). Tangible Bits: Beyond Pixels. Proceedings of the 2nd International Conference on Tangible and Embedded Interaction (pp. xvxxv). Bonn, Germany: New York, NY, ACM.

Jacob, J. K. R., Girouard, A., Hirshfield, M. L., Horn, S. M., Shaer, O., Solovey, T. E., \& Zigelbaum, J. (2007). Reality-Based interaction: Unifying the New Generation of Interaction Styles. CHI 2007 extended abstracts on Human Factors in computing systems (pp. 2465-2470). New York: ACM.

Jacob, J. K. R., Girouard, A., Hirshfield, M. L., Horn, S. M., Shaer, O., Solovey, T. E., \& Zigelbaum, J. (2008). Reality-Based interaction: A Framework for Post-WIMP Interface. Proceeding of the twenty-sixth annual SIGCHI conference on Human factors in computing systems (pp. 201210). New York:ACM.

Kaptelinin, V., \& Nardi, B. A. (2006). Acting with Technology: Activity Theory and Interaction Design. Cambridge, MA: The MIT Press.

Lakoff, G. (1987). Woman, fire and dangerous things: What categories reveal about the mind. Chicago: The University of Chicago Press.

Lakoff, G. \& Johnson, M. (1980). Metaphors we live by. Chicago:The University of Chicago Press.

Polanyi, M. (1966). The tacit dimension: The University of Chicago Press.

Lombard, M. \& Ditton, T. (1997). At the heart of it all: The concept of presence. Journal of Computer Mediated Communication, 3(2). Retrieved September, 2009, From http://jcmc.indiana.edu/vol3/issue2/lombard.html Riva, G. (2005). The Psychology of Ambient Intelligence: Activity, Situation and Presence. In G. Riva, F. Vatalaro \& F. Davide (Eds.), Ambient Intelligence: The Evolution of Technology,
Communication and Cognition (pp. 17-34). Amsterdam: IOS Press.

Reed, E. S. (1996). Encountering the World: Toward An Ecological Psychology. New York: Oxford University Press.

Rogers, Y., Scaife, M., Gabrielli, S., Smith, H., \& Harris, E. (2002). A Conceptual Framework for Mixed Reality Environments: Designing Novel Learning Activities for Young Children. PRESENCE Journals, 11(6), 677-686. MIT Press.

Waterworth, J. A. (1999). Spaces, Places, Landscapes, and Views: Experiential Design of Shred Information Spaces. A. In J. Munro, K. Höök \& D. Benyon (Eds.), Social Navigation of Information Space. London: Springer-Verlag.

Waterworth, J. A., Ballesteros, S., Peter, C., Bieber, G., Kreiner, A., Wiratanaya, A., Polymenakos, L., Wanche-Politis, S., Capobianco, M., Etxeberria, L., \& Lundholm, L. (2009). Ageing in a Networked Society, Social Inclusion and Mental Stimulation. Proceedings of the 2 nd International Conference on PErvsive Technologies Related to Assistive Environments. Corfu, Greece: New York, ACM.

Waterworth, J. A., Lund, A., \& Modjeska, D. (2003). Experiential Design of Shared Information Spaces. K. Höök, D. Benyon, \& A. J. Munro. (Eds.), Designing Information Spaces: The Social Navigation Approach (pp. 125-149). Great Britain: Springer.

Waterworth E. L. \& Waterworth, J. A. (2010). Mediated Presence in the Future, In C. C. Bracken, \& P. Skalski (Eds.), Immersed in Media: Telepresence in Everyday Life (pp. 183-196). New York: Routledge, Taylor \& Francis Group.

Ullmer, B., \& Ishii, H. (2000). Emerging frameworks for tangible user interfaces. IBM Systems Journal, 39(3), 915-931.

Zacks, T. Z., Hasher, L., \& Li, K. Z. H. (2000). Human Memory. In F. I. M. Craik \& T. A. Salthouse (Eds.), The Handbook of Aging and Cognition (pp. 293-358). Hillsdale, NJ: LEA. 